\title{
Suggestion of a new dimensionless number for dynamic plastic response of beams and plates
}

\author{
Y. P. Zhao
}

Summary A dimensionless number, termed response number in the present paper, is suggested for the dynamic plastic response of beams and plates made of rigid-perfectly plastic materials subjected to dynamic loading. It is obtained at dimensional reduction of the basic governing equations of beams and plates. The number is defined as the product of the Johnson's damage number and the square of the half of the slenderness ratio for a beam; the product of the damage number and the square of the half of the aspect ratio for a plate or membrane loaded dynamically. Response number can also be considered as the ratio of the inertia force at the impulsive loading to the plastic limit load of the structure. Three aspects are reflected in this dimensionless number: the inertia of the applied dynamic loading, the resistance ability of the material to the deformation caused by the loading and the geometrical influence of the structure on the dynamic response. For an impulsively loaded beam or plate, the final dimensionless deflection is solely dependent upon the response number. When the secondary effects of finite deflections, strain-rate sensitivity or transverse shear are taken into account, the response number is as useful as in the case of simple bending theory. Finally, the number is not only suitable to idealized dynamic loads but also applicable to dynamic loads of general shape.

Key words dynamic plastic response, structure, Johnson's damage number, response number

1

Introduction

For a continuum loaded impulsively, or impinged by an initial velocity pulse $V_{0}$, Johnson's damage number is defined by [1]

Dn $=\frac{\rho V_{0}^{2}}{\sigma_{0}}$,

where $\rho$ and $\sigma_{0}$ are density and yield stress of the material, respectively. Parameter Dn is a basic dimensionless similarity parameter in impact dynamics. In fact, the damage number can be obtained by reducing to dimensionless terms the equation of motion. As an example, consider the well-known equation of motion for a one-dimensional problem

$\frac{\partial \sigma}{\partial x}=\rho \frac{\partial V}{\partial t}$,

where $\sigma$ and $V$ denote stress and particle velocity, respectively. To make it dimensionless, we introduce the dimensionless variables as follows:

Received 17 October 1997; accepted for publication 19 March 1998

Ya-Pu Zhao

Laboratory for Nonlinear Mechanics of Continuous Media (LNM), Institute of Mechanics, Chinese Academy of Sciences, Beijing 100080, People's Republic of China

Fax: (86-10)62561284; E-mail: yzhao@lnm.imech.ac.cn

The supports given by the National Natural Science Foundation of China and the Foundation of director of the Institute of Mechanics, CAS, are gratefully acknowledged. 
$\Sigma=\frac{\sigma}{\sigma_{0}}, \tau=\frac{t}{T}, X=\frac{x}{V_{0} T}, \bar{V}=\frac{V}{V_{0}}$,

where $T$ is a characteristic time. By using transformation (3), we can render (2) dimensionless as

$\frac{\partial \Sigma}{\partial X}=\frac{\rho V_{0}^{2}}{\sigma_{0}} \frac{\partial \bar{V}}{\partial \tau}$

It is evident from (4) that damage number is a dominant dimensionless parameter for the dynamic plastic response of material. The damage number can be understood as a measure of the order of strain imposed in the region where severe plastic deformation occurs; it can also be considered the ratio of inertia force of the loading $\left(\rho V_{0}^{2}\right)$ to the resistance ability of the dynamically loaded material $\left(\sigma_{0}\right)$. In the same manner, Cauchy number [2-4]

$\mathrm{Ca}=\frac{\rho V_{0}^{2}}{E}$,

can be obtained by making dimensionless Eq. (2) through the introduction of a dimensionless stress $\Sigma=\sigma / E$, other dimensionless variables are the same as (3), where $E$ is the Young's modulus of the material. Cauchy number is thus an important similarity parameter for the dynamic elastic response of material under impact loading.

A rectangular pressure pulse $p(t)=p[H(t)-H(t-\tau)]$ represents an idealization for dynamic loads in the field of dynamic plasticity of structures $[3,5]$, where $p$ and $\tau$ are the magnitude and the duration of the pulse, respectively, $H(t)$ is the Heaviside step function. The damage number equivalent to (1) for this kind of loading is [6]

Dn $=\frac{I^{2}}{\rho \sigma_{0} H^{2}}$

where $I=p \tau$ is the impulse of the rectangular pressure pulse, $H$ is the thickness of a plate or a beam.

In practice, dynamic loads may be complicated, varying rapidly with time. They can not be described approximately and effectively by a velocity pulse or rectangular pressure pulse. Still, for dynamic loads of general shape, the damage number equivalent to (1) and (6) can be also defined as [7]

$\mathrm{Dn}=\frac{I_{e}^{2}}{\rho \sigma_{0} H^{2}}$

where

$I_{e}=\int_{t_{0}}^{t_{f}} p(t) \mathrm{d} t$

is the effective impulse of the applied loading, $t_{0}$ and $t_{f}$ are the times when plastic deformation begins and ends, while $t_{f}$ can be found as

$p_{0}\left(t_{f}-t_{0}\right)=I_{e}$,

where $p_{0}$ is the plastic limit load of the structure.

Generally speaking, two aspects are reflected in the damage number Dn, the inertia of the impulsive loading $\rho V_{0}^{2}$ and the resistance ability of the material to plastic deformation $\sigma_{0}$. These two aspects are the main characteristics of dynamic plastic response of materials. For dynamic plastic response of structures, besides the aforementioned two factors, there is another important factor, that is the influence of the geometry of the structure.

The objective of the present paper is to suggest, by making dimensionless the governing equations, a dimensionless number which can reflect the three factors of the dynamic plastic response of structures: inertia of the loading, the resistance ability of the material of the structure to deformation and the structural geometry. 


\section{Derivation at dimensionless governing equations of beams and plates}

It is well known that the Reynolds number Re in fluid dynamics can be obtained by making dimensionless the Navier-Stokes equations. Reynolds number $R e=\rho V L / \mu=V L / v$ represents the ratio of the inertia force $\rho V^{2}$ to the shear stress $\mu V / L$, where $v$ is the kinematic viscosity.

The derivation of Re is instructive for the study of the dynamic plastic response of structures subjected to impact loading. For simplicity and without loss of generality, we first consider a beam subjected to uniformly distributed impulsive loading. The governing equation of the beam is $[3,5]$

$m^{\prime}=\int_{0}^{x} \frac{\mu \ddot{w}}{M_{0}} \mathrm{~d} x-\frac{4 n}{H} w^{\prime}$

where $\mu$ is the mass of the beam per unit length, thus $\mu=\rho H, w$ is the transverse deflection of the beam, $m=M / M_{0}$ and $n=N / N_{0}$ are dimensionless bending moment and axial force, respectively. The quantity $M_{0}=\sigma_{0} H^{2} / 4$ denotes the fully plastic bending moment per unit length of a beam with a rectangular cross section and unit width, $N_{0}$ is the fully plastic axial force per unit length. The second term on the right-hand side of (9) introduces the axial forces arising from axial constraints. This term vanishes for simple bending theory. Differentiating (9) with respect to $x$ we obtain

$\frac{\partial^{2} m}{\partial x^{2}}=\frac{\mu \ddot{w}}{M_{0}}-\frac{4 n}{H} \frac{\partial^{2} w}{\partial x^{2}}-\frac{4}{H} \frac{\partial w}{\partial x} \frac{\partial n}{\partial x}$

If the beam span is $2 L$, and the dimensionless variables $X, W$ and $T$ are introduced through

$X=\frac{x}{L}, W=\frac{w}{H}, t=\frac{H T}{V_{0}}$,

then the dimensionless form of (9) is

$\frac{1}{4} \frac{\partial^{2} m}{\partial X^{2}}=\frac{\rho V_{0}^{2}}{\sigma_{0}}\left(\frac{L}{H}\right)^{2} \frac{\partial^{2} W}{\partial T^{2}}-n \frac{\partial^{2} W}{\partial X^{2}}-\frac{\partial W}{\partial X} \frac{\partial n}{\partial X}$

It is easy to see that the coefficient of the first term on the right-hand side of (11) is the dominant similarity parameter for dynamic plastic response of beams subjected to impulsive loading. For convenience, this dimensionless number is termed "response number" by the present paper as

$\mathrm{Rn}=\frac{\rho V_{0}^{2}}{\sigma_{0}}\left(\frac{L}{H}\right)^{2}$.

From (12) follows that response number is the product of the Johnson's damage number and the square of the half of the slenderness ratio of the beam. Three aspects are reflected in this number, i.e. the inertia of the load $\rho V_{0}^{2}$, the resistance ability of the material to plastic deformation $\sigma_{0}$, and the geometry of the structure $L / H$. Actually, the latter two factors could be combined in one, the plastic limit load of the beam. If the plastic limit load of a beam is of the order $p_{0} \sim \sigma_{0}(H / L)^{2}$, then the response number can be also considered of the order of the ratio of the inertia of the impulsive loading to the plastic limit load of the beam $p_{0}$, i.e.

$\mathrm{Rn} \sim \frac{\rho V_{0}^{2}}{p_{0}}$

Similarly, the response number for a rectangular pressure pulse can be expressed by

$\mathrm{Rn}=\frac{I^{2}}{\rho \sigma_{0} H^{2}}\left(\frac{L}{H}\right)^{2}$ 
For dynamic load of general shape, the response number is

$\mathrm{Rn}=\frac{I_{e}^{2}}{\rho \sigma_{0} H^{2}}\left(\frac{L}{H}\right)^{2}$.

For simplicity and without loss of generality, we take a circular plate as an example for the case of plates. If both rotatory inertia and axial force are disregarded, the motion equation of a plate subjected to impulsive loading in polar coordinates $(r, \theta)$ is $[3,10,11]$

$\left(r M_{r}\right)^{\prime}-M_{\theta}=\int_{0}^{r} m \ddot{w} r \mathrm{dr}$,

where $m$ is the mass of the plate per unit area of neutral plane, thus, $m=\rho H,(\cdot)^{\prime}=\partial(\cdot) / \partial r,(\cdot)=\partial(\cdot) / \partial t . M_{r}$ and $M_{\theta}$ are radial and circumferential bending moments per unit length, respectively. Differentiating (16) with respect to $r$, we have

$\left(r M_{r}\right)^{\prime \prime}-M_{\theta}^{\prime}=\rho H \ddot{w} r$.

If the radius and thickness of a circular plate are denoted by $L$ and $H$, respectively, and if we introduce $R$ as the dimensionless radial coordinate, $T$ as the dimensionless time, and $W$ as the dimensionless deflection, then we have the following relationship:

$R=\frac{r}{L}, t=\frac{H T}{V_{0}}, W=\frac{w}{H}$.

Letting $m_{R}=M_{r} / M_{0}$ and $m_{\theta}=M_{\theta} / M_{0}$ denote dimensionless radial and circumferential bending moments per unit length, respectively, we have the dimensionless motion equation of the circular plate as follows:

$\frac{1}{R} \frac{\partial^{2}\left(R m_{R}\right)}{\partial R^{2}}-\frac{1}{R} \frac{\partial m_{\theta}}{\partial R}=4 \frac{\rho V_{0}^{2}}{\sigma_{0}}\left(\frac{L}{H}\right)^{2} \frac{\partial^{2} W}{\partial T^{2}}$.

By comparing (19) and (11), it is easy to find that the same dimensionless parameter-damage number $\mathrm{Rn}=\left(\rho V_{0}^{2} / \sigma_{0}\right)(L / H)^{2}$ is also included in (19). For a circular plate, since the plastic limit carrying capacity is $p_{0} \sim \sigma_{0}(H / L)^{2}$, the response number for circular plate can also be considered as the ratio of the inertia of the load to the plastic limit carrying capacity.

In general, for the study of dynamic plastic response of beams and plates subjected to uniformly distributed impact loads, a quite useful dimensionless product can be put forward as

$\mathrm{Rn}=\operatorname{Dn}\left(\frac{L}{H}\right)^{2}$,

where $L$ and $H$ are two characteristic dimensions of the structures. For a simply supported (or fully clamped) beam, $L$ and $H$ are taken to be the semi-length and the thickness; for a circular plate or membrane, $L$ and $H$ are taken to be the radius and the thickness; for a simply supported (or fully clamped) square plate, $L$ and $H$ are taken to be the semi-width and the thickness; and for a rectangular plate, $L$ and $H$ are taken to be semi-width and thickness, respectively. From the definition of (20), we know that the dimensionless number $\mathrm{Rn}$ is the product of the damage number expressed in (1), (6) or (7) and the square of half the slenderness ratio for a beam, the product of the damage number and the square of half the aspect ratio for a circular plate or a membrane, for a square plate as well as for a rectangular plate.

\section{3}

Dimensionless expressions of the permanent displacements of some structures loaded dynamically

To show the validity of the damage number in the analysis of dynamic plastic response of structures, some known results will be related to this dimensionless number in this section. Besides the results of simple bending theory, some secondary effects such as finite- deflections, 
strain-rate sensitivity, transverse shear etc., will be also considered. The dynamic loads include idealized ones such as impulsive loading, rectangular pressure pulse, as well as dynamic loads of general shape.

To avoid ambiguity, we first define the two characteristic dimensions of a structure. The semi-span of the beam, the radius of the circular plate, and the half width of the rectangular plate will be denoted by $L$; the thickness of the aforementioned structures will be represented by $H$.

\section{1}

\section{Simply supported beam subjected to uniformly distributed impulsive loading $\boldsymbol{V}_{\mathbf{0}}$}

Consider a simply supported, rigid-perfectly plastic beam of unit width. By using the simplified Tresca yield conditions, the dimensionless maximum deflection at the midpoint of the beam is [12]

$\frac{w_{f}}{H}=\frac{1}{3} \frac{\mu V_{0}^{2} L^{2}}{M_{0} H}$

where $M_{0}=\sigma_{0} H^{2} / 4$ is the fully plastic bending moment of the beam per unit length, $\mu$ the mass of the beam per unit length. Equation (21) is only valid for infinitesimal displacements. If the second-order effects of finite displacements are considered, then the final central displacement of the beam is [13]

$\frac{w_{f}}{H}=\frac{1}{4}\left[\left(1+3 \frac{\mu V_{0}^{2} L^{2}}{M_{0} H}\right)^{1 / 2}-1\right]$.

If both bending moments and axial forces are considered, the dimensionless permanent midpoint displacement of the beam is [3]

$\frac{w_{f}}{H}=\frac{1}{4}\left\{\left[\frac{1}{2}+2 \frac{\mu V_{0}^{2} L^{2}}{M_{0} H}+\frac{1}{2}\left(1+\frac{8}{3} \frac{\mu V_{0}^{2} L^{2}}{M_{0} H}\right)^{1 / 2}\right]^{1 / 2}-1\right\}$.

By using the response number $\mathrm{Rn}$ in (12), Eqs. (21)-(23) can be recast into the form of

$$
\left\{\begin{array}{l}
\frac{w_{f}}{H}=\frac{4}{3} \mathrm{Rn}, \\
\frac{w_{f}}{H}=\frac{1}{4}\left[(1+12 \mathrm{Rn})^{1 / 2}-1\right], \\
\frac{w_{f}}{H}=\frac{1}{4}\left\{\left[\frac{1}{2}+8 \mathrm{Rn}+\frac{1}{2}\left(1+\frac{32}{3} \mathrm{Rn}\right)^{1 / 2}\right]^{1 / 2}-1\right\}
\end{array}\right.
$$

Relations (24) that the dimensionless central displacement of a beam subjected to uniformly distributed impulsive loading is dependent solely upon the response number, in other words, only upon the ratio of the inertia force of the loading to the plastic limit carrying capacity of the beam.

It should be noted that (21)-(23) are only valid when [3]

$\frac{Q_{0} L}{2 M_{0}} \geq 3 / 2$

where $Q_{0} \approx \sigma_{0} H / 2$ is the fully plastic transverse shear force per unit length of the rectangular cross section. For $1 \leq Q_{0} L /\left(2 M_{0}\right) \leq 3 / 2$, second-order effect of transverse shear must be considered. The central permanent displacement of the beam is changed from (21) to [3]

$w_{f}=\frac{\mu L^{2} V_{0}^{2}}{4 M_{0}} \frac{1+6(\lambda-1)}{4 \lambda-3}$, 
where $\lambda=Q_{0} L /\left(2 M_{0}\right)$. Obviously, (26) can be recast into the dimensionless form

$$
\frac{w_{f}}{H}=\operatorname{Rn} \frac{6 \lambda-5}{4 \lambda-3} \text {. }
$$

Evidently, (27) can be reduced to the first one of (24) when $\lambda=3 / 2$.

\section{2}

\section{Fully clamped beam subjected to uniformly distributed impulsive loading $\mathbf{V}_{\mathbf{0}}$}

The dimensionless permanent deflection of the mid-point of a fully clamped beam of unit width loaded impulsively is expressed in the form of [3]

$\frac{w_{f}}{H}=\frac{1}{2}\left[\left(1+\frac{3}{4} \frac{\mu V_{0}^{2} L^{2}}{M_{0} H}\right)^{1 / 2}-1\right]$.

By using the response number in (12), (28) can be changed into the following form:

$\frac{w_{f}}{H}=\frac{1}{2}\left[(1+3 \mathrm{Rn})^{1 / 2}-1\right]$.

Equation (28) is developed for a square yield curve that circumscribes the exact maximum normal stress-yield curve. The influence of finite-deflections is retained in the governing equations. Assume that the yield condition for bending moment $M$ and axial force $N$ is taken as

$\frac{|M|}{M_{0}}+\left(\frac{N}{N_{0}}\right)^{2}=1$

Then the dimensionless final displacement, assumed to be the maximum excursion of the midpoint, is found to be [13]

$\frac{w_{f}}{H}=\frac{1}{2}\left[\sqrt{1+\frac{2}{3} \frac{\mu V_{0}^{2} L^{2}}{M_{0} H}}-1\right]$.

Equation (30) can be obviously expressed in the form

$\frac{w_{f}}{H}=\frac{1}{2}\left(\sqrt{1+\frac{8}{3} \mathrm{Rn}}-1\right)$.

As the simply supported beam, the dimensionless final displacement of a fully clamped beam loaded impulsively is only dependent upon the ratio of the inertia force of the impulsively loading to the plastic limit carrying capacity of the beam.

It is well known that the yield stress of most metallic materials increases with strain rate. Cowper and Symonds suggested the following constitutive equation;

$\dot{\epsilon}=D\left(\frac{\sigma_{0}^{\prime}}{\sigma_{0}}-1\right)^{q}, \quad \sigma_{0}^{\prime} \geq \sigma_{0}$,

where $\sigma_{0}^{\prime}$ is the dynamic yield stress at an uniaxial plastic strain rate $\dot{\epsilon}, \sigma_{0}$ the associated static yield stress, $D$ and $q$ are constants for a particular material. Obviously, (32) can be recast into the form of

$\frac{\sigma_{0}^{\prime}}{\sigma_{0}}=1+\left(\frac{\dot{\epsilon}}{D}\right)^{\frac{1}{q}}$

For an impulsively loaded fully clamped beam of unit width, if strain-rate sensitivity is taken into account, then (29) can be changed into 


$$
\frac{w_{f}}{H}=\frac{1}{2}\left[\left(1+\frac{3}{n^{\prime}} \mathrm{Rn}\right)^{1 / 2}-1\right],
$$

where the ratio of the dynamic yield stress to the associated static yield stress is [3]

$n^{\prime}=\frac{\sigma_{0}^{\prime}}{\sigma_{0}}=1+\left(\frac{V_{0} w_{f}}{3 \sqrt{2} D L^{2}}\right)^{1 / q}$.

An experimental investigation [14] was conducted into the dynamic plastic response and failure of fully clamped metal beams which were subjected to uniformly distributed impulsive loading over the entire span (shown in Fig. 1). Three failure modes were classified in [14] for fully clamped beams with rectangular cross sections as follows:

Mode I: large inelastic deformation of the entire beam;

Mode II: tearing (tensile failure) of the beam material at the supports;

Mode III: transverse shear failure of the beam material at the supports.

These three failure modes are illustrated in Fig. 2.

On the basis of the result of the experiment [14], an approximate theoretical study [15] was carried out for predicting the onset of these three failure modes. For Mode I failure of fully

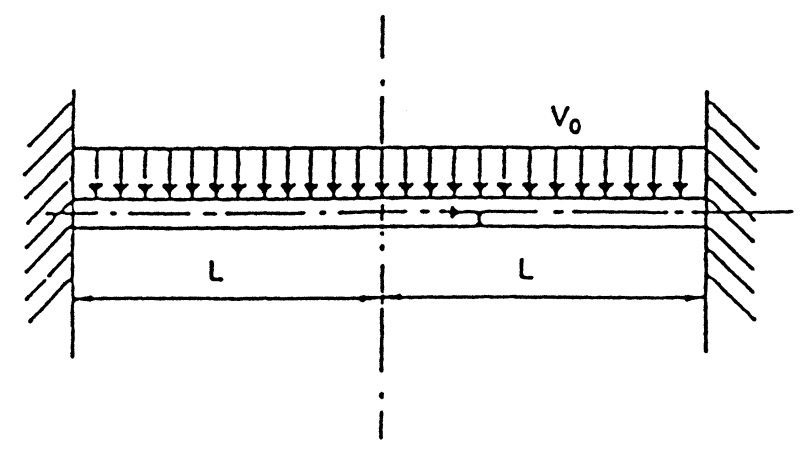

Fig. 1. Clamped beam subjected to impulsive loading
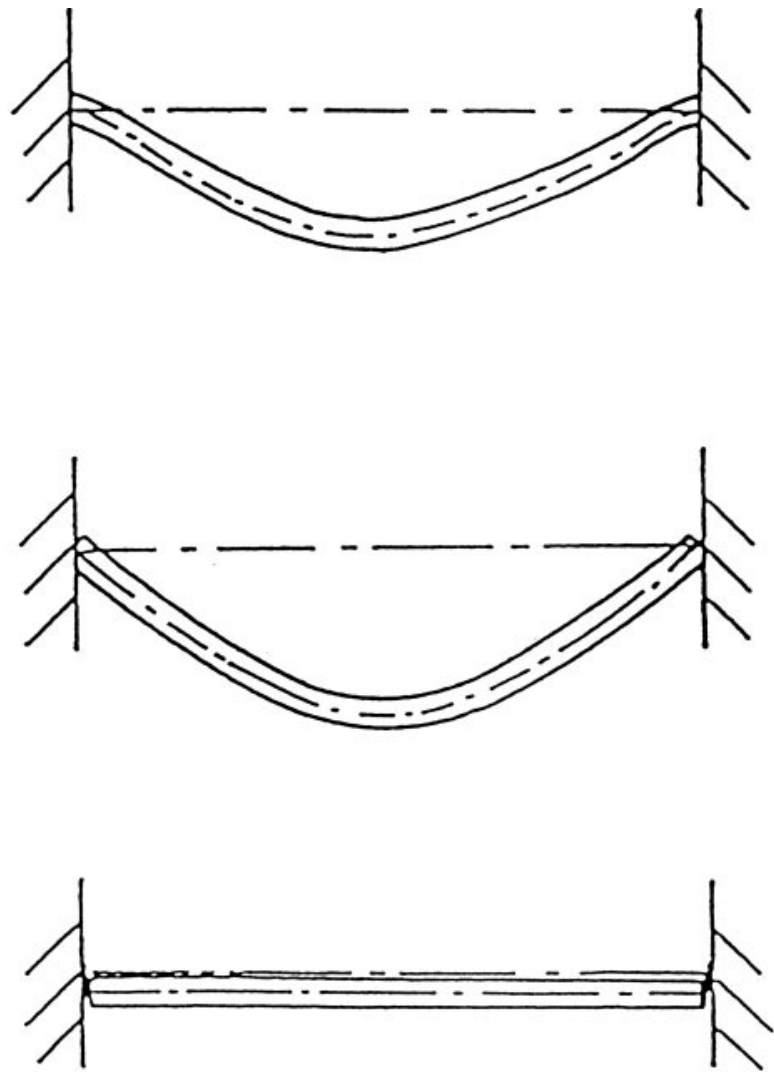

Fig. 2. Three typical failure modes of impulsively loaded clamped beam 
clamped, rigid-perfectly plastic beam with unit width, (28) or (29) can be used to predict the large plastic deformation. Mode II-failure is caused by excessive tensile strain, the threshold velocity to cause such kind of failure is given by

$V_{0}=\left\{1+\sqrt{2\left[2+\epsilon_{r}\left(2 \frac{L}{H}\right)^{2}-2 \frac{L}{H}\right]}\right\} \frac{H}{L} \sqrt{\frac{\sigma_{0}}{3 \rho}}$,

where $\epsilon_{r}$ is the critical value of the tensile strain. By using the dimensionless number Rn in (12), (34) can be rearranged into

$\mathrm{Rn}=\frac{1}{3}\left\{1+\sqrt{2\left[2+\epsilon_{r}\left(2 \frac{L}{H}\right)^{2}-2 \frac{L}{H}\right]}\right\}^{2}$.

3.3

Simply supported beam subjected to rectangular pressure pulse

If the beam is subjected to uniformly distributed rectangular pressure pulse, the relationship of the impulse with the impulsive loading is $I=p \tau=\rho V_{0} H$, then the dimensionless form of the beam's motion equation is

$\frac{1}{4} \frac{\partial^{2} m}{\partial X^{2}}=-\frac{p}{\sigma_{0}}\left(\frac{L}{H}\right)^{2}+\frac{I^{2}}{\rho \sigma_{0} H^{2}}\left(\frac{L}{H}\right)^{2} \frac{\partial^{2} W}{\partial T^{2}}-n \frac{\partial^{2} W}{\partial X^{2}}-\frac{\partial W}{\partial X} \frac{\partial n}{\partial X}$.

It is easy to see that the first term on the right-hand side of above equation is the order of the ratio of the magnitude of the load to the plastic limit load of the beam; thus, both the response number $\mathrm{Rn}$ and $p / p_{0}$ will be included in the expression of the final displacement.

If the beam is subjected to uniformly distributed rectangular pressure pulse in medium range $p_{0} \leq p \leq 3 p_{0}$, the final mid-point displacement of the beam is given by [3]

$w_{f}=3 \frac{p}{p_{0}}\left(\frac{p}{p_{0}}-1\right) \frac{M_{0} \tau^{2}}{2 \mu L^{2}}$,

where $p_{0}=2 M_{0} / L^{2}$ is the static collapse load of the beam. In the same manner as the impulsive loading, (37) can be rewritten into the following dimensionless form

$\frac{w_{f}}{H}=\frac{3}{2} \operatorname{Rn}\left(1-\frac{p_{0}}{p}\right)$,

where (14) is used in the expression of the response number $\mathrm{Rn}$.

Similarly, for high load $p>3 p_{0}$, the dimensionless permanent central displacement of the beam is

$\frac{w_{f}}{H}=\frac{4}{3} \mathrm{Rn}\left(1-\frac{3}{4} \frac{p_{0}}{p}\right)$.

By comparing the first equation in (24) and (39), we know that the result of impulsive loading is the limit case of that of the rectangular pressure pulse when $p_{0} / p \rightarrow 0$ and $\tau \rightarrow 0$.

\section{4}

Free-free beam

The dynamic plastic failure of a free-free beam was studied in [16]. The amplitude of the rotational motion of the mid-point of the free-free beam subjected to uniformly distributed rectangular pressure pulse is

$w_{f}=\frac{p \tau^{2}}{2 \mu}\left(\frac{p}{p_{0}}-1\right)$. 
By virtue of response number Rn, (40) can be expressed by a dimensionless form as

$$
\frac{w_{f}}{H}=\frac{\mathrm{Rn}}{6}\left(1-\frac{p_{0}}{p}\right),
$$

where (14) is used in the expression of the response number Rn. For impulsive loading, the limit of (41) when $p_{0} / p \rightarrow 0$ and $\tau \rightarrow 0$ is then

$$
\frac{w_{f}}{H}=\frac{\mathrm{Rn}}{6},
$$

where (12) is used in the expression of the response number Rn.

\section{5}

\section{Circular plate}

Consider a simply supported, rigid-perfectly plastic circular plate subjected to uniformly distributed rectangular pressure pulse. For a medium load $p_{0}<p<2 p_{0}$, the final displacement at the center of the plate is [17]

$w_{f}=\frac{p \tau^{2}\left(p-p_{0}\right)}{\mu p_{0}}$,

where $p_{0}=6 M_{0} / L^{2}$ is the static collapse load of the circular plate, the simplified Tresca yield condition has been used to obtain (43). By using the response number Rn in (14), (43) can be changed into the following dimensionless form

$\frac{w_{f}}{H}=\frac{2}{3} \mathrm{Rn}\left(1-\frac{p_{0}}{p}\right)$.

Similarly, for a high load $p \geq 2 p_{0}$, the dimensionless final displacement at the center of the plate is

$\frac{w_{f}}{H}=\frac{1}{3} \operatorname{Rn}\left(\frac{3}{2}-\frac{p_{0}}{p}\right)$.

The result for uniformly distributed impulsive loading is the limit case of (45) when $p_{0} / p \rightarrow 0$ and $\tau \rightarrow 0$ [18]; thus, the dimensionless final central displacement of the plate is

$\frac{w_{f}}{H}=\frac{\mathrm{Rn}}{2}$.

It should be noted that (46) is valid when [3]

$\frac{Q_{0} L}{2 M_{0}} \geq 2$.

For $3 / 2 \leq Q_{0} L /\left(2 M_{0}\right) \leq 2$, the effect of transverse shear must be considered, then central permanent displacement of the plate is [3]

$w_{f}=\frac{\mu V_{0}^{2} L^{2}}{24 M_{0}} \frac{4 \lambda-5}{\lambda-1}$,

where $\lambda=Q_{0} L /\left(2 M_{0}\right)$ for circular plate. Equation (48) can be rewritten into

$\frac{w_{f}}{H}=\frac{1}{6} \operatorname{Rn} \frac{4 \lambda-5}{\lambda-1}$.

It is obvious that (49) can be reduced to (46) when $\lambda=2$. In fact, $Q_{0} L /\left(2 M_{0}\right) \approx L / H$ holds for beam and plate; thus, this value can also be termed half the slenderness ratio or aspect ratio for 
a beam or a plate, respectively. If the second-order effect of transverse shear is considered, we know from both (27) and (49) that the dimensionless final central displacements of beam and plate are dependent upon two factors: the response number Rn and the slenderness or thinness ratio.

Consider a simply supported, rigid-perfectly plastic circular plate subjected to impulsive loading. If finite-deflections effect is taken into account, then the dimensionless permanent deflection of the center of the circular plate is given by [3]

$\frac{w_{f}}{H}=\frac{1}{2}\left[\left(1+\frac{2}{3} \frac{\mu V_{0}^{2} L^{2}}{M_{0} H}\right)^{1 / 2}-1\right]$.

By using the response number, (50) may be recast into the form of

$\frac{w_{f}}{H}=\frac{1}{2}\left[\left(1+\frac{8}{3} \mathrm{Rn}\right)^{\frac{1}{2}}-1\right]$.

A closed-form solution was obtained by [8] for the dynamic plastic deformation of a simplysupported circular plate subjected to a pressure pulse of general shape. This solution is also based on the simplified Tresca yield surface. The final central displacement of the plate for no hinge band $\left(p_{\max } \leq 2 p_{0}\right)$ is

$w_{f}=\frac{I_{e}^{2}}{\mu p_{0}}\left(1-\frac{p_{0}}{p_{e}}\right)$,

where $p_{e}=I_{e}^{2} / 2 \int_{t_{0}}^{t_{f}}\left(t-t_{0}\right) p(t) \mathrm{d} t$ is the effective pressure: Equation (52) can be changed into

$\frac{w_{f}}{H}=\frac{2}{3} \operatorname{Rn}\left(1-\frac{p_{0}}{p_{e}}\right)$.

It should be noted that (15) is used in the expression of the response number. The final displacement at the center of the plate with hinge band is found to be [8]

$w_{f}=\frac{I_{e}^{2}}{\mu p_{0}}\left[1-\frac{p_{0}}{p_{e}}-\frac{1}{2}\left(\frac{I_{e}^{*}}{I_{e}}\right)^{2}\left(\frac{1}{2}-\frac{p_{0}}{p_{e}^{*}}\right)\right]$,

where

$I_{e}^{*}=\int_{t_{0}}^{t_{c}} p(t) \mathrm{d} t, p_{e}^{*}=\frac{I_{e}^{* 2}}{2} \int_{t_{b}}^{t_{c}}\left(t-t_{b}\right) p(t) \mathrm{d} t$

$t_{b}$ is the time when $p(t)=2 p_{0}$, and $t_{c}$ is the time when the hinge band shrinks to the origin. Similarly, the dimensionless form of (54), by using the response number in (15), is

$\frac{w_{f}}{H}=\frac{2}{3} \mathrm{Rn}\left[1-\frac{p_{0}}{p_{e}}-\frac{1}{2}\left(\frac{I_{e}^{*}}{I_{e}}\right)^{2}\left(\frac{1}{2}-\frac{p_{0}}{p_{e}^{*}}\right)\right]$.

The result was known of the dynamic plastic response of a simply supported annular plate with inner radius $a$ and outer radius $L$ subjected to rectangular pressure pulse with a distribution $p(r)=p(L-r) /(r-a), a \leq r \leq L$ (see for example [3]). $r$ is the radial coordinate. The dimensionless permanent displacement distribution under impulsive loading which is the limit case of that under rectangular pressure pulse is given by

$\frac{W_{f}}{H}=\frac{\mu V_{0}^{2} L^{2}}{24 M_{0} H}(1+3 \alpha)\left(1-\frac{r}{L}\right)$,

where $\alpha=a / L$. It is obvious that (56) can be rewritten into the form of 
$\frac{W_{f}}{H}=\frac{\mathrm{Rn}}{6}(1+3 \alpha)\left(1-\frac{r}{L}\right)$

The behavior of a rigid-perfectly plastic circular plate was studied in [19]. The plate is fully clamped around the outer boundary and subjected to impulsive loading. The dimensionless maximum transverse displacement, which occurs at the plate center, is approximately

$\frac{w_{f}}{H} \cong 0.84 \frac{\mu V_{0}^{2} L^{2}}{12 M_{0} H}$

Equation (58) may be changed into the form of

$\frac{w_{f}}{H} \cong 0.28 \mathrm{Rn}$

The dynamic behavior of a fully clamped circular plate subjected to rectangular pressure pulse was studied in [20]. The permanent central deflection of the plate is

$w_{f}=\varphi \frac{I^{2} L^{2}}{\mu M_{0}}$

where $\varphi=\varphi\left(p / p_{0}\right)$ is a function of the ratio of the magnitude of the applied load to the plastic limit load of the plate. Equation (60) may be changed into the form of

$\frac{w_{f}}{H}=4 \varphi \mathrm{Rn}$.

\section{6}

Circular membrane

The dimensionless final displacement at the center of a circular membrane is [3]

$\frac{w_{f}}{H}=\left(\frac{2}{3} \frac{\rho V_{0}^{2}}{\sigma_{0}} \frac{L^{2}}{H^{2}}\right)^{1 / 2}$.

It is obvious that (62) can be rearranged into the following form:

$\frac{w_{f}}{H}=\sqrt{\frac{2}{3} \mathrm{Rn}}$.

The behavior of an impulsively loaded circular plates was examined in [21], and a more exact theoretical membrane mode solution was obtained, which can be written in the form

$\frac{w_{f}}{H}=1.0186\left(\frac{2}{3} \frac{\rho V_{0}^{2}}{\sigma_{0}} \frac{L^{2}}{H^{2}}\right)^{1 / 2}$

i.e.

$\frac{w_{f}}{H}=1.0186 \sqrt{\frac{2}{3} \mathrm{Rn}}$.

If the strain-rate effect is considered, (63) is changed into

$\frac{w_{f}}{H}=\left(\frac{2}{3 n^{\prime}} \mathrm{Rn}\right)^{1 / 2}$,

where 
$n^{\prime}=\frac{\sigma_{0}^{\prime}}{\sigma_{0}}=1+\left[\frac{V_{0} w_{f}}{3 \sqrt{2} D L^{2}}\right]^{1 / q}$

\section{7}

\section{Square and rectangular plates}

The dynamic response was theoretically investigated in [22] for a simply supported, rigidperfectly plastic square plate, side length $2 L$ and thickness $H$, subjected to rectangular pressure pulse. It is interesting to note that the results in this case are the same as those of a simply supported circular plate, namely, the dimensionless final displacement at the center of the square plate is also represented by (44) for medium load $p_{0}<p<2 p_{0}$, and (45) still holds for the high load $p \geq 2 p_{0}$.

The dimensionless permanent central displacement of a fully clamped square plate with side length $2 L$ and thickness $H$ under uniformly distributed impulsive loading is [3]

$\frac{w_{f}}{H}=\sqrt{1+\frac{2}{3} \frac{\rho V_{0}^{2} L^{2}}{\sigma_{0} H^{2}}}-1$

Thus, (67) is rewritten into the form of

$\frac{w_{f}}{H}=\sqrt{1+\frac{2}{3} \mathrm{Rn}}-1$

The maximum dimensionless deflection of a fully clamped rectangular plate with semi-width $L$ and semi-length $L^{\prime}$ subjected to impulsive loading is [23]

$\frac{w_{m}}{H}=\frac{\left(3-\xi_{0}\right)\left\{\left[1+\frac{\mu V_{0}^{2} L^{2}}{6 M_{0} H}\left(3-2 \xi_{0}\right)\left(1-\xi_{0}+\frac{1}{2-\xi_{0}}\right)\right]^{1 / 2}-1\right\}}{2\left[1+\left(\xi_{0}-1\right)\left(\xi_{0}-2\right)\right]}$,

where

$\xi_{0}=\frac{L}{L^{\prime}} \tan \phi, \quad \tan \phi=-\frac{L}{L^{\prime}}+\sqrt{3+\left(\frac{L}{L^{\prime}}\right)^{2}}$

It is obvious that (69) may be rewritten into the form of

$\frac{w_{m}}{H}=\frac{\left(3-\xi_{0}\right)\left\{\left[1+\frac{2}{3} \operatorname{Rn}\left(3-2 \xi_{0}\right)\left(1-\xi_{0}+\frac{1}{2-\xi_{0}}\right)\right]^{1 / 2}-1\right\}}{2\left[1+\left(\xi_{0}-1\right)\left(\xi_{0}-2\right)\right]}$.

It is noted that $\xi_{0}=1$ for square plate. In this case it is easy to see that (69) or (70) can be reduced to (67) or (68). It should be also noted (70) can be reduced to the result of fully clamped beam (29) when $\xi_{0}=0$.

In the same manner as outlined above, the dimensionless maximum deflection of a simplysupported rectangular plate with semi-width $L$ and semi-length $L^{\prime}$ subjected to impulsive loading may be written as

$\frac{w_{m}}{H}=\frac{\left(3-\xi_{0}\right)\left\{\left[1+\frac{8}{3} \operatorname{Rn}\left(3-2 \xi_{0}\right)\left(1-\xi_{0}+\frac{1}{2-\xi_{0}}\right)\right]^{1 / 2}-1\right\}}{4\left[1+\left(\xi_{0}-1\right)\left(\xi_{0}-2\right)\right]}$.

By substituting $\xi_{0}=1$, we can easily get the result for simply-supported square plate or simplysupported circular plate (51). By substituting $\xi_{0}=0$, we can obtain the result of simplysupported beam, which is expressed by the second equation in (24). 
For a fully clamped rectangular plate, if the effect of strain-rate sensitivity is considered, then the expression of theoretical dimensionless central displacement is changed from (70) to the following form:

$$
\frac{w_{m}}{H}=\frac{\left(3-\xi_{0}\right)\left\{\left[1+\frac{2}{3 n^{\prime}} \operatorname{Rn}\left(3-2 \xi_{0}\right)\left(1-\xi_{0}+\frac{1}{2-\xi_{0}}\right)\right]^{1 / 2}-1\right\}}{2\left[1+\left(\xi_{0}-1\right)\left(\xi_{0}-2\right)\right]},
$$

where $n^{\prime}$ is given in Eq. (66). The results for a fully clamped square plate and bearn can be obtained by substituting $\xi_{0}=1$ and $\xi_{0}=0$ into above equation, respectively.

\section{4}

\section{Discussion and conclusions}

A dimensionless response number $\mathrm{Rn}$ is suggested herein in order to study the dynamic plastic response and failure of structures under impulsive loading. The number is obtained by introducing dimensionless equations of motion of beams and plates. From (11) and (19) it follows that response number is the only dimensionless similarity parameter for the dynamic plastic response of beam and plate under impulsive loading. It is not only suitable for any boundary conditions (simply supported, fully clamped, etc.) and loading conditions (impulsive loading, rectangular pressure pulse, dynamic loads of general shape) but also describes secondorder effects such as finite deflections, transverse shear, strain-rate sensitivity as well as dynamic tearing.

In order to show its ability to predict the dynamic behavior of structures under dynamic loading, Fig. 3 illustrates the use of $\mathrm{Rn}$ for comparison of the experimental results with simple rigid-plasic models, which retain finite-deflection effects for rectangular plate with aspect ratio 0.593 [3]. By using Rn, Fig. 4 shows the comparison between experimental and
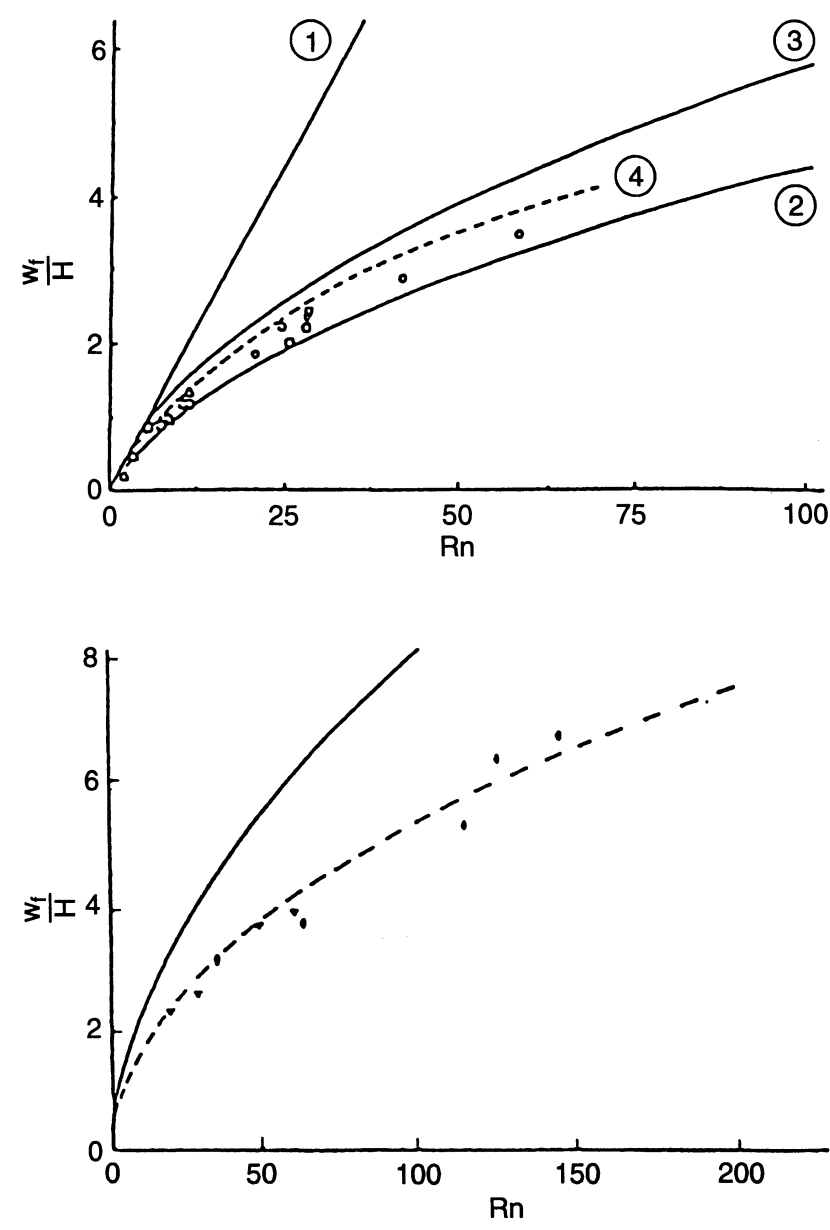

Fig. 3. Use of Rn to compare the experimental results with simple rigidplastic models for rectangular plate with aspect ratio $0.593[3](\bigcirc, \square, \triangle)$ experimental results on aluminium 6061 T6 rectangular plate; 1 approximate infinitesimal analysis for a rigid-perfectly plastic material; 2,3 bounds from a rigidperfectly plastic analysis with finite deflections; 4 finite-deflection analysis with an exact yield surface for a rigid-perfectly plastic material

Fig. 4. Use of $\mathrm{Rn}$ to compare the experimental results for mild steel beams with theoretical ones [3] ( $\bullet$ ) experimental results (-) rigid-perfectly plastic analysis for a strain-rate-insensitive material, including finite-deflection effects; (---) rigid-perfectly plastic analysis for a strain-rate sensitive material, including finite-deflection effects 
theoretical results for beams, which retain finite-deflection and strain-rate sensitivity effects [3].

It is necessary to point out that the beam discussed in the present paper is with unit width. If the width of the beam is $B$, the final central displacements given above still hold, in this case $\mu=\rho H B$, and the fully plastic bending moment is changed from $M_{0}=\sigma_{0} H^{2} / 4$ to $M_{0}=\sigma_{0} B H^{2} / 4$.

Is the method suggested in this paper instructive for dynamic elastic response of structures? The answer is positive, Cauchy number in (5) for this case will be used instead of damage number. For example, the dynamic elastic buckling condition of a spherical shell [24] subjected to uniformly distributed exterior impulsive loading can be expressed consistently to the form of response number as

$\frac{\rho V_{0}^{2}}{E}\left(\frac{R}{H}\right)^{2}=\frac{2}{(1+v)(1-v)}$,

where $v$ is Poisson's ratio, $R$ and $H$ are average radius and the thickness of the thin shell. The dimensionless number can be termed the elastic response number $\mathrm{Rn}_{e}=C a(R / H)^{2}$, thus, the dynamic elastic buckling condition of a spherical shell can be understood when the elastic response number reaches a critical value. For example, if the Poisson's ratio of the shell material is $v=0.3$, then the elastic dynamic buckling condition can be written as

$\mathrm{Rn}_{e}=2.20$

\section{References}

1. Johnson, W.: Impact strength of materials. Edward Arnold 1972

2. Johnson, W.; Sengupta, A. K.; Ghosh, S. K.; Reid, S. R.: Mechanics of high speed impact at normal incidence between plasticine long rods and plates. J. Mech. Phys. Solids 29 (1981) 413-445

3. Jones, N.: Structural impact. Cambridge: University Press 1989

4. Zhao, Y. P.: On some dimensionless numbers in fracture mechanics. Int. J. Fracture 83 (1997) L7-L13

5. Zhao, Y. P.; Yu, T. X.; Fang, J.: Large dynamic plastic deflection of a simply supported beam subjected to rectangular pressure pulse. Arch. Appl. Mech. 64 (1994) 223-232

6. Nurick, G. N.; Martin, J. B.: Deformation of thin plates subjected to impulsive loading - A review. Part II: Experimental studies. Int. J. Impact Eng. 8 (1989) 171-186

7. Zhao, Y. P.: Prediction of structural dynamic plastic shear failure by Johnson's damage number. Forsch Ingenieurwes. 63 (1998) 349-352

8. Youngdahl, C. K.: Influence of pulse shape on the final plastic deformation of a circular plate. Int. J. Solids Struct. 7 (1971) 1127-1142

9. Schlichting, H.: Boundary-layer theory, 7th ed., McGraw-Hill: Book Company 1979

10. Zhao, Y. P.; Fang, J.; Yu, T. X.: Dynamic plastic shear failure analysis for an infinitely large plate with a centred cylinder under impulsive loading. Int. J. Solids Struct. 31 (1994) 1585-1595

11. Zhao, Y. P.; Yu, T. X.; Fang, J.: Dynamic plastic behaviour of overhanging circular plate with various support conditions. Eng. Trans. 41 (1993) 297-309

12. Jones, N.: Influence of strain-hardening and strain-rate sensitivity on the permanent deformation of impulsively loaded rigid-plastic beams. Int. J. Mech. Sci. 9 (1967) 777-796

13. Symonds, P. S.; Jones, N.: Impulsive loading of fully clamped beams with finite plastic deflections and strain-rate sensitivity. Int. J. Mech. Sci. 14 (1972) 49-69

14. Menkes, S. B.; Opat, H. G.: Broken beams. Exp. Mech. 13 (1973) 480-486

15. Jones, N.: Plastic failure of ductile beams loaded dynamically. J. Eng. Indust 98 (1976) 131-136

16. Jones, N.; Wierzbicki, T.: Dynamic plastic failure of free-free beam. Int. J. Impact Eng. 6 (1987) 225240

17. Hopkins, H. G.; Prager, W.: On the dynamics of plastic circular plates. Z. Angew. Math. Phys. 5 (1954) 317-330

18. Wang, A. J.: The permanent deflection of a plastic plate under blast loading. J. Appl. Mech. 22 (1955) 375-376

19. Wang, A. J.; Hopkins, H. G.: On the plastic deformation of built-in circular plates under impulsive load. J. Mech. Phys. Solids 3 (1954) 22-37

20. Florence, A. L.: Clamped circular rigid-plastic plates under blast loading. J. Appl. Mech. 33 (1966) 256260

21. Symonds, P. S.; Wierzbicki, T.: Membrane mode solutions for impulsively loaded circular plate. J. Appl. Mech. 46 (1979), 58-64

22. Cox, A. D.; Morland, L. W.: Dynamic plastic deformation of simply supported square plates. J. Mech. Phys. Solids 7 (1959) 229-241 
23. Jones, N.: A theoretical study of the dynamic plastic behavior of beams and plates with finite-deflections. Int. J. Solids Struct. 7 (1971) 1007-1029

24. Jones, N.; Ahn, C. S.: Dynamic elastic and plastic buckling of complete spherical shells. Int. J. Solids Struct. 10 (1974) 1357-1374 